\title{
Influencia de los factores sedentarios (dieta y videojuegos) sobre la obesidad en escolares de Educación Primaria
}

Gabriel González Valero. Universidad de Granada

Félix Zurita Ortega. Universidad de Granada

Pilar Puertas Molero. Universidad de Granada

Tamara Espejo Garcés. Universidad de Granada

Ramón Chacón Cuberos. Universidad de Granada

Manuel Castro Sánchez. Universidad de Granada

Recepción: 16 de febrero de 2017 | Aceptado: 14 de marzo de 2017

Correspondencia: Gabriel González Valero | Correo-e: gabrielgonzalezvalero@gmail.com

\author{
iD 0000-0001-7472-5694 \\ Citar: Gonzalez, G., Zurita, F., Puertas, P., Espejo, T., Chacon, R. y Castro, M. (2017). \\ Influencia de los factores sedentarios (dieta y videojuegos) sobre la obesodad en escolares de \\ Educación Primaria. ReiDoCrea, 6, 120-129.
}

\begin{abstract}
Resumen: El primer ciclo de la etapa de Educación Primaria es un periodo en el que se comienza con la configuración de hábitos saludables de cara a la adolescencia donde se ponen de manifiesto que los hábitos alimentarios se encuentran en decadencia y los problemas de sobrepeso y obesidad van en aumento paulatino, influenciados por las prácticas digitales sedentarias. La finalidad de este estudio es la de definir los índices de masa corporal, la adherencia a la dieta mediterránea y uso problemático de videojuegos y establecer relaciones entre si y el género. En una muestra de 79 sujetos con edades comprendidas de 6 a 8 años $(M=7,1$; D.T. $=0.638)$ se utilizó un diseño de carácter descriptivo, comparativo y de corte transversal, donde se utilizaron instrumentos como la báscula, test KIDMED y test CERV. Los resultados evidencian la necesidad de mejorar la dieta, reeducando los hábitos alimentarios y promover la actividad física para reducir los problemas de obesidad y sobrepeso.
\end{abstract}

Palabras Clave: Hábitos Saludables | Educación Primaria

Influence of the Sedentary Factors (diet and video games) on Obesity in Students of the Primary Education

\begin{abstract}
Primary Education is a period in which we begin to shape healthy habits in preparation for adolescence where it is revealed that eating habits decline and the problems of overweight and obesity gradually increase, influenced by sedentary digital practices. The purpose of this study is to define body mass indexes, adherence to the Mediterranean diet and the problematic use of video games and establish relationships between them and gender. A descriptive, comparative and cross-sectional methodological design was used in a sample of 79 subjects aged 6 to 8 years $(M=7.1$; $D T=0.638)$, using the KIDMED test and CERV test. The results demonstrate the need to improve diet, re-educate about eating habits and promote physical activity to reduce problems of obesity and overweight.
\end{abstract}

Keywords: Healthy Habits | Primary Education

\section{Introducción}

Los hábitos saludables en la niñez y la adolescencia, son un punto esencial para alcanzar el desarrollo máximo en el ámbito físico e intelectual, pero estudios epidemiológicos muestran que los cambios en los hábitos alimentarios y tecnológicos producidos en los últimos años, han provocado un alarmante aumento de niños y niñas con problemas derivados del sedentarismo (Ruiz-Pons, García-Nieto, González, Mérida, Valenzuela y Aguirre-Jaime, 2008).

Por ello, la creación de hábitos saludables en edades tempranas es un factor clave para mejorar el estado de salud del alumnado, así como lo es asentar una base en el 
alumnado, para que en su etapa de vida adulta se continúe desarrollando un estilo de vida saludable (Rosell y Alfonso, 2014). Los cambios sociales experimentados, dan lugar a una trasformación, en lo que se refiere a problemas y necesidades a nivel de salud y de educación. En 1998, la World Health Organization (WHO) en la Fifty-first World Health Assembly, enunciaba que la salud es uno de los derechos fundamentales de los seres humanos (Guilbert, 2003). Gozar de ella y del bienestar depende de la interrelación de factores, entre los que el estilo de vida es el más importante (Religioni y Czerw, 2012).

La salud y calidad de vida ocupan un lugar central en la sociedad, puesto que constituye un derecho, valor, aspiración o recurso. En conexión con lo citado, el concepto de obesidad y sobrepeso es un desafío implacable (Zylke y Bauchner, 2016). Como dice Moscetti y Pronk (2017), es un tema muy tratado, ya que, se ha convertido en una de las enfermedades emergentes del siglo XXI y se le ha catalogado como la Epidemia Global del siglo XXI. Son múltiples las definiciones, pero la gran mayoría inciden en que es la acumulación o el exceso de grasa en el organismo resultante del desbalance entre ingestas calóricas y la eliminación de las mismas (Saavedra y Dattilo, 2012; Li et al.,2016 y Cheung, Cunningham, Narayan, y Kramer 2016).

Teniendo en cuenta lo comentado, se ha calculado que aproximadamente el $10 \%$ de los jóvenes en edad escolar (entre 5 y 17 años) del mundo, tiene exceso de grasa corporal y una cuarta parte de ellos son obesos (Kumanyika, Jeffery, Morabia, Ritenbaugh y Antipatis, 2002). Tal y como argumentan Rodríguez et al. (2016), la obesidad infantil ha aumentado de forma considerable en los países desarrollados debido a cambios en el estilo de vida, priorizándose hábitos más sedentarios y a insuficientes e inadecuadas ingestas. En España, las cifras del Eurobarómetro 2009 muestran que la práctica de la actividad física y deportiva se encuentra por debajo de la media europea y con un elevado índice de sedentarismo, siendo uno de los países europeos con mayor prevalencia de obesidad y sobrepeso infantil con un $26.3 \%$ en edades entre 2 y 24 años (Rosell y Alfonso, 2014).

Al hilo de lo comentado, se destacan estudios donde el ocio sedentario como un estilo de vida donde el entretenimiento se basa en la utilización de instrumentos tecnológicos o ver la televisión (Martínez-Gómez, Eisenmann, Gómez-Martínez, Veses, Marcos y Veiga 2010 y Chacón et al., 2016). Las tecnologías de la información y comunicación como internet, se han transformado en una adicción entre los más jóvenes (Acier y Kern, 2009). De ahí los problemas de obesidad infantil, que se encuentran íntimamente ligados al sedentarismo existente y a las tecnologías que nos rodean (Daley, 2009 y Onis, Blössner y Borghi, 2010). Es así como, los niveles de ocio digital representan otro de los elementos que pueden influir en el nivel de adherencia en la dieta mediterránea, pues constituyen hábitos sedentarios que generalmente se asocian a un estilo de vida menos saludable con dietas más irregulares (Falbe, Willett, Rosner, Gortmaker, Sonneville y Field, 2014). Añadir, que gracias a la industria del videojuego, se ha creado una tecnología muy novedosa capaz de mapear el esqueleto humano, registrando cada uno de sus movimientos en tiempo real para trasladarlos a la pantalla, conocidos como "Exergames" o "Videojuegos activos" (Trujillo, Muñoz y Villalda, 2013 y Castro-Sánchez, Espejo-Garcés, Valdivia-Moral, Zurita-Ortega, Chacón-Cuberos y Cabrera-Fernández, 2015).

Los primeros estudios científicos sobre los efectos psicosociales de los videojuegos comenzaron a publicarse a principios de la década de los 80 , abarcando áreas como la personalidad, la salud o la coordinación óculo-manual (Anderson, Gentile y Buckley, 2007). Herman, Sabiston, Mathieu, Tremblay y Paradis (2014) y Ruiz y Castañeda 
(2016), son autores que nos hablan de que actualmente, pasar demasiadas horas frente a las pantallas digitales disminuye el tiempo destinado a la práctica de ocio, actividad física y relaciones sociales. En este sentido, el uso problemático de videojuegos o las horas frente a las pantallas, se encuentran estrechamente ligados con factores psicosociales y la calidad de vida, ya que es evidente que tienen un gran potencial adictivo, son inductores de conductas violentas y agresivas entre los jóvenes, convierten a las personas en seres socialmente aislados y producen efectos negativos sobre el rendimiento escolar, debido al absentismo y a un posible desplazamiento del tiempo de estudio (Salguero, del Río y Vallecillo, 2009; Hastings, Karas, Winsler, Way, Madigan y Tyler, 2009; Chaín-Pinzón y Briñez, 2011 y Montes, Bozal, Torres y Núñez, 2016).

La práctica regular de actividad físico-deportiva se considera un elemento esencial e imprescindible para una buena salud, así como para conseguir un crecimiento óptimo y desarrollo en escolares (Trost y Loprinzi, 2008). Los estudios explican la importancia de la práctica de actividad física, a la hora de producir mejora a nivel físico, psíquico y social, al igual que la calidad de vida (Moliner-Urdiales et al., 2009 y Khan, Chu, Kirk y Veugelers, 2012). De este modo, se ha construido en las últimas décadas el concepto de actividad física entendida como el conjunto de conductas motrices llevadas a cabo durante el tiempo libre o la vida cotidiana que implican gasto energético, mayor que el que una persona tiene cuando está en reposo (Eime, Young, Harvey, Charity y Payne, 2013).

Del mismo modo, la actividad física es de vital importancia a la hora de la prevención de enfermedades como las patologías cardiovasculares, reducción del índice de grasa corporal y problemas asociados a los estilos de vida sedentarios (Eisenmann, 2004; Chen, Lio, Cook, Bass y Lo, 2009). De hecho estas premisas indican que el alumnado confiere un valor a su cuerpo, lo acepta con sus limitaciones y se incrementa las prácticas físico-saludables (Guillamón, Cantó, Soto y García, 2016; Colin y Martínez 2016). Muchos de los hábitos que se adquieren en esta etapa se repetirán durante el resto de la vida adulta, lo cual, justifica la importancia de estudio e implementación de hábitos saludables en edad escolar (Becerra, Pinzón y Vargas, 2011 y García, Sellés y Cejuela, 2015).

Dicha importancia, radica en describir y relacionar los hábitos físico-saludables y sedentarios de una muestra de escolares de 6 a 8 años, siendo éste, un ámbito de estudio poco explorado y muy repercusivo en edades posteriores. Así, según lo establecido con anterioridad, los principales objetivos que persigue este estudio son los de establecer los niveles de índices de masa corporal, el grado de adherencia a la dieta mediterránea y el uso problemático de video juegos en una muestra de estudiantes de Educación Primaria y determinar las posibles relaciones entre todos los parámetros y el género.

\section{Materiales y métodos}

\section{Diseño y Participantes}

Participaron en esta investigación de carácter descriptivo, comparativo y de corte transversal un total de 79 escolares de Educación Primaria de Granada (España), representando 34 al género femenino $(43 \%)$ y 45 al masculino $(57 \%)$, cuya edad estuvo comprendida entre los 6 y 8 años de edad $(7,1 \pm 0.638)$. Para este estudio, el método de muestreo es de carácter no probabilístico y por conveniencia. 


\section{Variables e instrumentos}

Las variables e instrumentos utilizados en este estudio fueron los siguientes:

- Género, según sea masculino o femenino, utilizando un cuestionario ad-hoc.

- Adhesión a la Dieta Mediterránea, se valoró el nivel de adhesión a la DM mediante el cuestionario KIDMED (Serrá-Majem et al., 2004). Este test cuenta con 16 ítems de tipo dicotómico de respuesta afirmativa o negativa, los cuales hacen referencia a patrones asociados al modelo mediterráneo. Cuatro de los ítems poseen connotación negativa $(-1)$, mientras que los doce restantes se valoran de forma positiva $(+1)$, oscilando la puntuación final entre $-4 y+12$. En el análisis descriptivo, el nivel de adherencia a la Dieta Mediterránea queda categorizado de la siguiente manera (Baja de -4 a 0; Media = de 1 a 6; Alta = de 7 a 12). Para el estudio correlacional se emplea la puntación total de la sumatoria de todos los ítems, este cuestionario se ha empleado en estudios similares como el de Chacón et al. (2016) o el de Muros, Cofre, Arriscado, Zurita y Knox (2016).

- Índice de Masa Corporal, Para calcular del Índice de Masa Corporal (IMC) se divide el peso (en kilogramos) por la talla (en metros) al cuadrado. Para determinar el peso se empleó el modelo de báscula de bioimpedancia y la cinta métrica Seca 206. Una vez obtenido el dato numérico, se utiliza la clasificación del estudio ENKID (Serrá-Majem y Aranceta-Bartrina, 2000), determinando cuatro niveles (Bajo Peso, Normopeso, Sobrepeso y Obesidad), para el estudio descriptivo. Para el estudio correlacional utilizo el valor real del IMC sin categorizar.

- Uso problemático de videojuegos, se valoró mediante el cuestionario CERV (Chamarro et al., 2014). Este cuestionario se compone de 17 ítems de connotación negativa, puntuados mediante una escala de tipo Likert de cuatro opciones ( $a=$ Casi nunca, $b=$ Algunas veces, $c=$ Bastantes veces, $d=$ Casi siempre); asignadas con puntuaciones del 1 al 4 respectivamente categorizando a los sujetos (Sin problemas: de 17 a 25; Problemas potenciales: de 26 a 38; Problemas severos: de 39 a 68). Para el análisis correlacional de la variable se realiza una sumatoria con los ítems con el fin de no perder potencia estadística. Se obtiene una fiabilidad de $\alpha=.872$.

\section{Procedimiento}

Se solicitó el acceso a los datos y consecución de la autorización de los organismos competentes, así como, la colaboración de los escolares participantes, todos ellos estudiantes del primer ciclo de Educación Primaria de la localidad de Granada, a través de una carta informativa elaborada para sus responsables legales. Posteriormente, los colegios que aceptaron participar en dicho estudio procedieron a informar a los padres, madres y tutores-as legales del alumnado, comunicándoles el procedimiento a realizar y su naturaleza, asegurándoles el anonimato de la misma, y evidentemente, poder obtener su consentimiento informado. La recogida de los datos, cabe destacar que se realizó en horario escolar y el proceso se llevó a cabo sin incidencias, siempre con la presencia de los investigadores, lo cuales iban leyendo pregunta a pregunta a los participantes para que se realizase una correcta aplicación de los instrumentos descritos, así como la resolución de dudas. Se debe señalar que se ha respetado el derecho de confidencialidad de todos los participantes y se eliminaron 31 cuestionarios por no hallarse debidamente cumplimentado. 


\section{Análisis de datos}

El análisis estadístico de datos fue analizado mediante el Paquete Estadístico para las Ciencias Sociales (SPSS 22.0), obtenidos datos que se han almacenado en archivo con extensión ".sav". En el estudio estadístico de los parámetros descriptivos y comparativos se emplean frecuencias y tablas cruzadas o de contingencia.

\section{Resultados}

De la muestra total (Tabla I), se han clasificados en su mayoría con un IMC de normopeso $(75,9 \%)$, le siguen los sujetos con obesidad $(13,9 \%)$ y sobrepeso $(8,9 \%)$ y solamente un $1,3 \%$ tienen bajo peso. Asimismo de los datos extraídos de la adherencia a la dieta mediterránea del total, el 40,5\% necesitan mejorar su patrón alimentario para ajustarlo al modelo mediterráneo y el $59,5 \%$ presentan una dieta óptima. En la variable videojuegos de ocio sedentario, el $96,2 \%$ no presentan problemas, exceptuando al $3,8 \%$ que manifestó problemas potenciales con los videojuegos.

\begin{tabular}{|c|c|c|c|}
\hline \multirow{2}{*}{\multicolumn{4}{|c|}{ Tabla I. Descriptivos de las variables de estudio }} \\
\hline & & & \\
\hline \multicolumn{2}{|l|}{ Hombres } & \multicolumn{2}{|l|}{$\mathrm{N}=45(57 \%)$} \\
\hline \multicolumn{2}{|l|}{ Mujeres } & \multicolumn{2}{|l|}{$\mathrm{N}=34(43 \%)$} \\
\hline \multicolumn{2}{|l|}{ IMC } & \multicolumn{2}{|l|}{ Adherencia DM } \\
\hline Bajo peso & $\mathrm{N}=1(1,3 \%)$ & \multirow{2}{*}{ Óptima } & \multirow{2}{*}{$\mathrm{N}=47(59,5 \%)$} \\
\hline Normopeso & $\mathrm{N}=60(75,9 \%)$ & & \\
\hline Sobrepeso & $\mathrm{N}=7(8,9 \%)$ & \multirow[t]{2}{*}{ Necesita mejorar } & \multirow[t]{2}{*}{$\mathrm{N}=32(40,5 \%)$} \\
\hline Obesidad & $\mathrm{N}=11(13,9 \%)$ & & \\
\hline \multicolumn{4}{|c|}{ Uso Problemático de Videojuegos } \\
\hline \multicolumn{2}{|c|}{$\begin{array}{ll}\text { Sin Problemas } \quad \mathrm{N}=7\end{array}$} & \multirow{2}{*}{$\begin{array}{l}=76(96,2 \%) \\
=3(3,8 \%)\end{array}$} & \\
\hline \multicolumn{2}{|c|}{ Problemas Potenciales } & & \\
\hline
\end{tabular}

Los resultados comparativos de las variables objeto de estudio se presentan en la tabla II. Se establecen las frecuencias comparativas en función del género, el IMC, uso de videojuegos y dieta mediterránea, donde no se detectan diferencias estadísticamente significativas entre los parámetros estudiados, ya que se obtiene una significatividad de $p>0,05$. Existe una tendencia a encontrar porcentajes elevados tanto de niños y niñas con problemas de sobrepeso y obesidad, donde casi la mitad de los sujetos de ambos géneros necesitan mejorar su patrón dietético. En el género masculino se comienzan a detectar los primeros problemas con los videojuegos.

\begin{tabular}{|c|c|c|c|c|}
\hline & & \multicolumn{3}{|l|}{ Género } \\
\hline & & Masculino ( $\mathrm{N}=45)$ & Femenino $(\mathrm{N}=34)$ & Sig. \\
\hline \multirow[t]{4}{*}{ IMC } & Bajo peso & $100 \%(n=1)$ & $0,0 \%(n=0)$ & \multirow[t]{4}{*}{0,154} \\
\hline & Normopeso & $61,7 \%(n=37)$ & $38,3 \%(n=23)$ & \\
\hline & Sobrepeso & $57,1 \%(n=4)$ & $42,9 \%(n=3)$ & \\
\hline & Obesidad & $27,3 \%(n=3)$ & $72,7 \%(n=8)$ & \\
\hline \multirow[t]{2}{*}{ CERV } & Sin Problemas & $55,3 \%(n=42)$ & $44,7 \%(n=34)$ & \multirow{4}{*}{$\begin{array}{l}0,125 \\
0,135\end{array}$} \\
\hline & Problemas Potenciales & $100 \%(n=3)$ & $0,0 \%(n=0)$ & \\
\hline \multirow[t]{2}{*}{ DIETA } & Necesita mejorar & $46,9 \%(n=15)$ & $53,1 \%(n=17)$ & \\
\hline & Óptima & $63,8 \%(n=30)$ & $36,2 \%(n=17)$ & \\
\hline
\end{tabular}


En la tabla III se expone la distribución entre la variable CERV, IMC y la adherencia a la dieta mediterránea. La tabla no revela datos estadísticos significativos siendo $p=0,291$ para el IMC y $p=0,796$ para la dieta mediterránea.

\begin{tabular}{|c|c|c|c|c|}
\hline \multicolumn{5}{|c|}{ CERV } \\
\hline & & Sin Problemas $(N=76)$ & Problemas Potenciales $(\mathrm{N}=3$ ) & Sig. \\
\hline \multirow[t]{4}{*}{ IMC } & Bajo peso & $100 \%(n=1)$ & $0,0 \%(n=0)$ & \multirow[t]{4}{*}{0,291} \\
\hline & Normopeso & $98,3 \%(n=59)$ & $1,7 \%(n=1)$ & \\
\hline & Sobrepeso & $85,7 \%(n=6)$ & $14,3 \%(n=1)$ & \\
\hline & Obesidad & $90,9 \%(n=10)$ & $9,1 \%(n=1)$ & \\
\hline \multirow[t]{2}{*}{ DIETA } & Necesita mejorar & $96,9 \%(n=31)$ & $3,1 \%(n=1)$ & \multirow[t]{2}{*}{0,796} \\
\hline & Óptima & $95,7 \%(n=45)$ & $4,3 \%(n=2)$ & \\
\hline
\end{tabular}

\section{Discusión}

Tal y como se estableció inicialmente, uno de los objetivos básicos de esta investigación es el de establecer los niveles de índices de masa corporal, el grado de adherencia a la dieta mediterránea y el uso problemático de video juegos en una población de niño y niñas en edad escolar, así como determinar las posibles relaciones entre todos los parámetros y el género. De hecho, el fin de estas premisas no es otro que el de revelar información sobre factores sedentarios para determinar la necesidad de intervenir o no, con programas físico-saludables. De este modo, encontramos algunos estudios similares a esta que aportan datos novedosos en cuanto grado de obesidad, práctica de actividad física y uso problemático de videojuegos (Sween, Wallington, Sheppard, Taylor, Llanos y Adams-Campbell, 2013 y Chacón, Zurita, Castro, Espejo, Martínez y Linares, 2016), además también existen investigaciones que aportan datos sobre hábitos saludables en escolares y población adolescente (Navarro-Solera, González-Carrascosa, y Soriano, 2014; San Mauro et al., 2015; Solís, Martín, Caro, Tomás, Menéndez y Galán, 2015 y Chacón et al., 2016).

Este estudio realizado en estudiantes granadinos de Educación Primaria aporta datos recientes sobre el índice de masa corporal, la adherencia a la dieta mediterránea y el uso problemático de videojuegos. En primer lugar, los datos indican que una cuarta parte de los sujetos participantes muestran índices de sobrepeso y obesidad, siendo el normopeso como el dato más común, abarcando tres cuartas partes de la población. Según las cifras del estudio ENKid realizado por Serrá-Majem y Aranceta-Bartrina (2000), revela que en España el 13,9\% presenta problemas de obesidad, mientras que si se combina este problema con el de sobrepeso, asciende hasta el $26,3 \%$ de la población estudiada. Por otro lado, el estudio realizado por Bacardí, Jiménez, Jones y Guzmán (2007), expone los datos de una muestra de 967 alumnos con edades comprendidas entre los 6 y 12 años, mostrando que un $21,1 \%$ de los participantes tienen sobrepeso, y un $23,5 \%$ son obesos. Sánchez, Jiménez, Fernández y Sánchez (2013), muestran datos semejantes, aportando que un $26 \%$ tienen sobrepeso, así como un $12,6 \%$ para los obesos (con un $38,6 \%$ total). Si comparamos resultados, podemos observar que según este estudio, dos niños (aproximadamente) por cada diez sufren exceso de peso, mientras que para el último estudio se obtienen cifras cercanas a los cuatro por cada diez.

Por otro lado, los descriptivos ofrecen que casi la mitad de los sujetos necesitan mejorar la dieta, mientras que el resto obtienen datos óptimo respecto a una adecuada dieta mediterránea. Lo que corrobora que casi la mitad de los participantes tienen que adecuar su patrón alimentario mediterráneo. Los datos que aportan Navarro-Solera, 
González-Carrascosa y Soriano (2014), hacen alusión a que un $46,7 \%$ de los participantes de su estudio presentan una dieta de media-baja calidad frente al $53,3 \%$ de dieta alta, de este modo se verifica tal necesidad de mejorar la dieta y su adherencia. Además se observan resultados similares en los estudio realizad por Falbe et al., (2014), en la adolescencia, donde aproximadamente dos tercios de los participantes siguen manteniendo un porcentaje medio de adherencia a la dieta mediterránea.

El análisis de las experiencias relacionadas con videojuegos, arroja datos donde algunos casos, presentaba problemas potenciales con los videojuegos; contrastando resultados con el estudio realizado por Chamarro et al., (2014), se pueden observar porcentajes inferiores, pues encontraron que siete de cada diez participantes padecía problemas potenciales, mientras que un $6 \%$ de la muestra eran severos. Esta variación se explica principalmente por diferencias de edad, ya que en la etapa primaria existe un mayor control parental y es en la adolescencia donde se muestra una mayor adicción hacia los videojuegos, observándose una disminución progresiva y lineal en participantes de diez a doce años y al aumentar la edad de los sujetos adolescentes con problemas potenciales (Van Rooij, Schoenmakers, Vermulst, Van Den Eijnden y Van De Mheen, 2011 y Granic, Lobel y Engels, 2014).

En edades tempranas no existe un uso problemático de videojuegos, pero existe una asociación donde a medida que el uso de los videojuegos es problemático se produce el aumento de los niveles de obesidad y sobrepeso, donde que un cuarto de los participantes que muestran un uso abusivo de las nuevas tecnología (Ruiz y Castañeda, 2016). Del mismo modo, cuanto más tiempo invierten los participantes en algún tipo de ocio sedentario, menos tiempo dedican a la actividad física, por tanto más problemas de sobrepeso y sedentarismo (Vázquez, Zapico, Díez y Rodríguez, 2008). Se aprecian casos donde el uso problemático de videojuegos (práctica sedentaria), se asocia con empobrecimiento del patrón alimentario mediterráneo (Manouchehri-Naeeni, 2015). En el estudio de Chacón et al. (2016), no se encontró correspondencia entre la adherencia a la DM y el nivel de adicción de videojuegos, pero el ocio sedentario produce problemas de sobrepeso y obesidad, en parte, por una dieta empobrecida (Chacón, Espejo, Cabrera, Castro, López y Zurita, 2015). Al igual que se detecta en este estudio, Muros, Cofre-Bolados, Salvador-Pérez, CastroSánchez, Valdivia-Moral y Pérez-Cortés (2016), asocian que a más horas frente a la pantalla menores valores de adherencia a la dieta mediterránea.

Por último, resaltar que el presente trabajo aborda conceptos que son actualmente de gran interés en el ámbito educativo. Por ello, a raíz de los datos e información extraída sobre factores sedentarios, invitamos a continuar estas líneas de trabajo a través de investigaciones con muestras más amplias, donde las variables se relacionen entre si y mediante programas de intervención físico-saludables, donde se puedan establecer las posibles diferencias y eficacia del programa en un determinado tiempo, de esta forma si se detecta un problema se solucionará. Añadir que de forma paralela, se podría canalizar el uso problemático de los videojuegos y factores sedentarios mediante programas de "Exergames".

\section{Conclusiones}

El estudio concluye que una cuarta parte de los participantes presentan problemas de sobrepeso y obesidad, mientras que las tres cuartas tienen un peso normal. Se extrae que casi la mitad del alumnado registrado necesita mejorar sus hábitos alimentarios y adherencia a la dieta mediterránea, esta necesidad de mejorar la dieta, pone de 
manifiesto reeducar los hábitos alimentarios con herramientas físico-saludables. El uso problemático de videojuegos se encuentra presente en uno de cada diez participantes.

Aun no encontrando asociación estadísticamente significativa entre las variables de estudio y el género de los participantes, se tiende a encontrar casos en el que los sujetos de esta investigación, se corresponden con los datos resultantes de los estudios encontrados durante el proceso de revisión bibliográfica.

Referencias

Acier, D., \& Kern, L. (2009). Problematic internet use: perceptions of addiction and chronic diseases in the United States. Journal of Public Health, 31, 496-505.

Anderson, C., Gentile, D., \& Buckley, K.E. (2007). Violent video game effects on children and adolescents: Theory, research, and public policy. Oxford: University Press.

Bacardí, M., Jiménez, A., Jones, E. y Guzmán, V. (2007). Alta prevalencia de obesidad y obesidad abdominal en niños escolares entre 6 y 12 años de edad. Bol Med Hosp Infant Mex, 64(6), 362-369.

Becerra, F., Pinzón, G. y Vargas, M. (2011). Hacia la creación del programa universidad promotora de la salud desde la alimentación y nutrición en la Universidad Nacional de Colombia, sede Bogotá. Revista de la Facultad de Medicina, 59(1), 67-76

Castro-Sánchez, M., Espejo-Garcés, T., Valdivia-Moral, P., ZuritaOrtega, F., Chacón-cuberos, R. y Cabrera-Fernández, A. (2015). Importancia de los exergames en la educación FísicoDeportiva. Revista de Transmisión del conocimiento Educativo y de la Salud, 7(5), 657-676.

Chacón, R., Castro, M., Muros, J., Espejo, T., Zurita, F., y Linares, M. (2016). Adhesión a la dieta mediterránea en estudiantes universitarios y su relación con los hábitos de ocio digital. Nutrición Hospitalaria, 33(2), 405-410.

Chacón, R., Espejo, T., Cabrera, Á., Castro, M., López, J. F. y Zurita, F. (2015) "Exergames" para la mejora de la salud en niños y niñas en edad escolar: estudio a partir de hábitos sedentarios e índices de obesidad/«Exergames» to improve the health of school children: study of sedentary lifestyle and obesity rates. Revista Latinoamericana de Tecnología Educativa, 14(2), 39-50.

Chacón, R., Zurita, F., Castro, M., Espejo, T., Martínez, A. y Linares, M. (2016). Estudio sobre la aplicabilidad de exergames para la mejora de los índices de obesidad y la imagen corporal en escolares. Revista Iberoamericana de Psicología del Ejercicio y el Deporte, 11(1), 97-105.

Chaín-Pinzón, N. y Briñez, B. (2011). Actividad física en adolescentes y su relación con agresividad, impulsividad, Internet y videojuegos. Psychologia, 5(1), 9-23.

Chamarro, A., Carbonell, X., Manresa, J., Muñoz-Miralles, R., Ortega-González, R., López-Morrón, M. y Torán-Monserrat, P. (2014). El Cuestionario de Experiencias Relacionadas con los Videojuegos (CERV): Un instrumento para detectar el uso problemático de videojuegos en adolescentes españoles. Adicciones, 26(4), 303-311.
Chen, S., Lio, M., Cook, J., Bass, S. y Lo, S. (2009). Sedentary lifestyle as a risk factor for low back pain: a systematic review. International Archives of Occupational and Enviromental, 82(7), 797-806.

Cheung, P., Cunningham, S., Narayan, K. y Kramer, M. (2016). Childhood obesity incidence in the United States: a systematic review. Childhood Obesity, 12(1), 1-11.

Colin, A. y Martínez, A. (2016). IMC en estudiantes de primer grado de primaria y su relación con la autoestima y el autoconcepto. PsicoEducativa, 2(3), 37-39.

Daley, A.J. (2009). Can exergaming contribute to improving physical activity levels and health outcomes in children? Pediatrics, 124(2), 763-771.

Eime, R., Young, J., Harvey, J., Charity, M., \& Payne, W. (2013). A systematic review of the psychological and social benefits of participation in sport for children and adolescents: informing development of a conceptual model of health through sport. International Journal of Behavioral Nutrition and Physical Activity, 10(1), 98.

Eisenmann, J. (2004). Physical activity and cardiovascular disease risk factors in children and adolescents: an overview. The Canadian journal of cardiology, 20(3), 295-301.

Falbe, J., Willett, W., Rosner, B., Gortmaker, S., Sonneville, K., \& Field, A. (2014). Longitudinal relations of television, electronic games, and digital versatile discs with changes in diet in adolescents. The American journal of clinical nutrition, 100(4), 1173-1181.

García, A., Sellés, S. y Cejuela, R. (2015). Estrategias de gestión y dirección para prevenir y paliar la obesidad infantil desde el ámbito educativo. Sportis, 1(1), 16-34.

Granic, I., Lobel, A., \& Engels, R. (2014). The benefits of playing video games. American Psychologist, 69(1), 66.

Guilbert, J. (2003). The world health report 2002-reducing risks, promoting healthy life. Education for health, 16(2), 230-230.

Guillamón, A., Cantó, E., Soto, J. y García, P. (2016). Estado de peso, condición física y satisfacción con la vida en escolares de educación primaria. Estudio piloto. MHSALUD: Revista en Ciencias del Movimiento Humano y Salud, 13(2), 1-15.

Hastings, E., Karas, T., Winsler, A., Way, E., Madigan, A., \& Tyler, S. (2009). Young children's video/computer game use: relations with school performance and behavior. Issues in mental health nursing, 30(10), 638-649. 
Herman, K., Sabiston, C., Mathieu, M., Tremblay, A. y Paradis, G. (2014). Sedentary behavior in a cohort of 8-to 10-year-old children at elevated risk of obesity. Preventive medicine, 60, 115-120.

Khan, M., Chu, Y., Kirk, S., \& Veugelers, P. (2015). Are sleep duration and sleep quality associated with diet quality, physical activity, and body weight status? A population-based study of Canadian children. Journal Public Health, 106(5), 277-282.

Kumanyika, S., Jeffery, R., Morabia, A., Ritenbaugh, C., \& Antipatis, V. (2002). Obesity prevention: the case for action. International journal of obesity, 26(3), 425.

Li, L., Yin, J., Cheng, H., Wang, Y., Gao, S., Li, M., \& Li, M. (2016). Identification of genetic and environmental factors predicting metabolically healthy obesity in children: Data from the BCAMS study. The Journal of Clinical Endocrinology and Metabolism, 101(4), 1816-1825.

Manouchehri-Naeeni, M., Jafari, S., Fouladgar, M., Heidari, K., Farajzadegan, Z., Fakhri, M., \& Omidi, R. (2014). Nutritional knowledge, practice, and dietary habits among school children and adolescents. International journal of preventive medicine, 5(2), 171.

Martínez-Gómez, D., Eisenmann, J.C., Gómez-Martínez, S., Veses, A., Marcos, A. y Veiga, O. (2010). Sedentarismo, adiposidad y factores de riesgo cardiovascular en adolescentes. Estudio AFINOS. Revista española de cardiología, 63(3), 277-285.

Moliner-Urdiales, D., Ruiz, J., Ortega, F., Rey-López, J., VicenteRodriguez, G., \& España-Romero, V. (2009). Association of objetively assessed physical activity with total and central body fat in Spanish adolescents. International Journal of Obesity, 33(10), 1126-1135.

Montes, J. C., Bozal, R., Torres, N. y Núñez, J. (2016). Consumo de nuevas tecnologías y factores de personalidad en estudiantes universitarios. Revista de comunicación y ciudadanía digital, 5(2), 203-228.

Moscetti, C., \& Pronk, N. (2017). Invisible seams: Preventing childhood obesity through an improved obstetrics-pediatrics care continuum. Preventive Medicine Reports, 5, 1-3.

Muros, J., Cofre-Bolados, C., Arriscado, D., Zurita, F., \& Knox, E. (2016). Mediterranean diet adherence is associated with lifestyles, physical fitness and mental wellness among 10-yearolds, in Chile. Nutrition, 35, 87-92.

Muros, J., Cofre-Bolados, C., Salvador-Pérez, S., Castro-Sánchez, M., Valdivia-Moral, P. y Pérez-Cortés, A. (2016). Relación entre nivel de actividad física y composición corporal en escolares de Santiago (Chile). Journal of Sport and Health Research, 8(1), 65-74.

Navarro-Solera, M., González-Carrascosa, R. y Soriano, J. M. (2014). Estudio del estado nutricional de estudiantes de educación primaria y secundaria de la provincia de Valencia y su relación con la adherencia a la Dieta Mediterránea. Revista Española de Nutrición Humana y Dietética, 18(2), 81-88.

Onis, M., Blössner, M. y Borghi, E. (2010). Prevalencia y las tendencias de sobrepeso y obesidad entre los niños en edad preescolar global. Diario Americano de la Nutrición Clínica, 92(5), 1257-1264.
Religioni, U. y Czerw, A. (2012). Health promotion in the context of National Health Programme to 2015. Progress in Health Sciences, 2(2), 167-173.

Rodríguez, G., Benito, P. J., Casajús, J., Ara, I., Aznar, S., Castillo, M. J. y Gracia-Marco, L. (2016). Actividad física, ejercicio y deporte en la lucha contra la obesidad infantil y juvenil. Nutrición Hospitalaria, 33(9), 1-21.

Rosell, L. y Alfonso, M.Á. (2014). Educación Física y promoción de la salud: estrategias de intervención en la escuela. Retos: nuevas tendencias en educación física, deporte y recreación, 25, 186-191.

Ruiz, R. y Castañeda, M. (2016). Relación entre uso de las nuevas tecnologías y sobrepeso infantil, como problema de salud pública. Enfermería Comunitaria, 4(1), 46-51.

Ruiz, R. y Castañeda, M. A. (2016). Relación entre uso de las nuevas tecnologías y sobrepeso infantil, como problema de salud pública. Enfermería Comunitaria, 4(1), 46-51.

Ruiz-Pons, M., García-Nieto, V., González, M., Mérida, G., Valenzuela C., \& Aguirre-Jaime, A. (2008). Reduced nocturnal systolic blood pressure dip in obese children. Nefrología, 28(5), 517-524.

Saavedra, J. y Dattilo, A. (2012). Factores alimentarios y dietéticos asociados a la obesidad infantil: recomendaciones para su prevención antes de los dos años de vida. Revista Peruana de Medicina Experimental y Salud Pública, 29(3), 379-385.

Salguero, R., del Río, M. y Vallecillo, J. (2009). Efectos psicosociales de los videojuegos. Revista Internacional de Comunicación Audiovisual, Publicidad y Estudios Culturales, 7 , 235-250.

San Mauro, I., Megías, A., de Angulo, B. G., Bodega, P., Rodríguez, P., Grande, G. y Garicano, E. (2015). Influencia de hábitos saludables en el estado ponderal de niños y adolescentes en edad escolar. Nutrición Hospitalaria, 31(5), 1996-2005.

Sánchez, J., Jiménez J., Fernández, F., \& Sánchez, M. (2013). Prevalence of child and youth obesity in Spain in 2012. Revista Española de Cardiología, 66(5), 371-376.

Serra-Majem, L. y Aranceta-Bartrina, J. (2000). Desayuno y equilibrio alimentario. Estudio enKid. Editorial Masson.

Serra-Majem, L., Ribas, L., Ngo, J., Ortega, R. M., García, A., Pérez-Rodrigo, C., \& Aranceta, J. (2004). Food, youth and the Mediterranean diet in Spain. Development of KIDMED, Mediterranean Diet Quality Index in children and adolescents. Public health nutrition, 7(7), 931-935.

Solís, D., Martín, J. D., Caro, F., Tomás, I., Menéndez, E. y Galán, I. (2015). Efectividad de una intervención escolar contra la obesidad. Anales de Pediatría, 83(1), 19-25.

Sween, J., Wallington, S. F., Sheppard, V., Taylor, T., Llanos, A., \& Adams-Campbell, L. (2013). The role of exergaming in improving physical activity. Journal of Physical Activity and Health, 11(4), 864-870.

Trost, S., \& Loprinzi, P. (2008). Exercise-Promoting healthy lifestyles in children and adolescent. Journal of clinical lipidology, 2(3), 162-168. 
Trujillo, J. Muñoz, J. y Villada, J. (2013). Exergames: una herramienta tecnológica para la actividad física. Revista Médica de Risaralda, 19(2), 126-130.

Van Rooij, A.J., Schoenmakers, T.M., Vermulst, A.A., Van Den Eijnden, R.J., \& Van De Mheen, D. (2011). Online video game addiction: identification of addicted adolescent gamers. Addiction, 106(1), 205-212.
Vázquez, I., Zapico, R., Díez, J. y Rodríguez, C. (2008). Actividad física, ocio sedentario, falta de sueño y sobrepeso infantil. Psicothema, 20(4), 516-520.

Zylke, J., \& Bauchner, H. (2016). The unrelenting challenge of obesity. Jama, 315(21), 2277-2278.

Revisión por pares abierta | Open Peer Review

Dr. Pedro Valdivia Moral. Universidad de Granada (Spain)

(iD) 0000-0002-1905-3247

Dr. Alfonso Conde Lacárcel. Universidad de Granada (Spain)

(iD $0000-0002-2247-2856$

Dra. Karen McMullin. Trent University (Canada)

(iD $0000-0002-1449-3550$ 